\title{
ESTUDIOS DE PLANTAS MEDICINALES PUBLICADOS POR LA ACADEMIA DE CIENCIAS MEDICAS, FISICAS Y NATURALES DE LA HABANA DE 1899 A 1958
}

\section{Mercedes Valero González}

La Academia de Ciencias de La Habana durante el siglo xx fue escenario de la presentación y discusión de trabajos sobre plantas medicinales, como continuación del interés que sobre esta temática se había desarrollado en el siglo pasado, cuando como bien expresó el académico doctor Manuel Mencía:

$$
\begin{aligned}
& \text {... no se podía ver con indiferencia que una rama tan ligada a la Medicina } \\
& \text { y a la Terapéutica como es la Botánica, no se utilizara debidamente entre } \\
& \text { nosotros, y que contando Cuba con una Flora Prodigiosa, y por lo tanto, } \\
& \text { con un vasto campo para la investigación sus plantas medicinales perma- } \\
& \text { necieran poco menos que ignoradas (1). }
\end{aligned}
$$

Los estudios desarrollados en esta línea durante el siglo xIX, en los cuales participaron destacadas personalidades de esta institución, perseguían entre sus objetivos fundamentales, comprobar las propiedades terapéuticas de las plantas del país, con el fin de conformar la flora médica y encontrar la base científica que diera respuesta al uso empírico de las drogas vegetales. Como resultado, en este nuevo período estudiado - siglo 
$\mathrm{xx}$ - fueron desarrollados y publicados una serie de trabajos, que a diferencia de anteriores, se caracterizaron por la utilización de análisis químicoorgánicos y la realización de ensayos sobre animales y personas.

Estas investigaciones, en las que participaron prestigiosas figuras científicas, como Juan Santos Fernández, Gastón Alonso Cuadrado e Ignacio Noble Xiqués, entre otros, recibieron una calurosa acogida en la institución, ya que contribuían a la aplicación de la «medicina verde» a partir de bases científicas y al mismo tiempo propiciaban la incorporación, de manera oficial, de los medicamentos de origen vegetal en la terapéutica cubana. El desarrollo de estos estudios estuvo condicionado, en la mayor parte de los casos, por el caudal de conocimientos empíricos que tenía la población sobre plantas que constituían elementos tradicionales significativos y que, dado su interés, fueron analizadas y estudiadas.

De los trabajos examinados, uno de los primeros que aportó datos sobre la utilidad médica de las plantas, fue el presentado por el doctor Jorge Le Roy y Cassá en 1904. Este estudio no perseguía en particular ahondar en las propiedades terapéuticas de la piña (Ananas comosus L.), sino en la presentación de un ejemplar con características monstruosas; no obstante, Le Roy aprovechó para indicar, entre los usos generales de la planta, sus aplicaciones médicas, para lo cual se apoyó en los datos aportados en sus obras por los médicos franceses Grossourdy, Baillon y Littré, quienes según el autor, afirman que los componentes fundamentales que se encuentran en esta fruta son el ácido cítrico y málico, azúcar, goma, albúmina, sustancias aromáticas y jalea. Además, Le Roy hace referencia a la utilización del jugo mezclado con azúcar y agua para las afecciones catarrales inflamatorias de las vías aéreas, urinarias e intestinales y lo definió como refrescante y antipútrido.

Otra de las propiedades médicas que le atribuye es la aplicación con buenos resultados en la litiasis renal de origen úrico (cálculos), en las dispepsias, enfermedades biliosas y en la hidropesía acompañada de íctero, así como en la utilización que hacían los prácticos de Estados Unidos de la piña de Cuba, como antídoto de los efectos provocados por la continuada ingestión de bebidas alcohólicas (2).

A pesar de su carácter teórico y de no constituir el resultado de un estudio químico experimental, el trabajo ofrece datos de interés sobre las propiedades terapéuticas que se atribuían a esta planta.

En 1908, el doctor José A. Fernández Benítez, químico y farmacéutico de la sección de ciencias de la Academia, presentó un pormenorizado estudio titulado Estudio fisiológico, químico y toxicológico del jugo del árbol del manzanillo, memoria laureada con el premio de medicina legal 
Doctor Antonio de Gordon y Acosta, y que tenía como objetivo fundamental contribuir al conocimiento y divulgación de las propiedades tóxicas de Mancinella venenata Tussac, o Hippomane mancinella Lin.

Al parecer, Fernández Benítez eligió esta planta por los serios peligros que entrañaba su uso y además porque, como señalaba el propio autor, "goza de propiedades verdaderamente extraordinarias» (3). De acuerdo con este trabajo, el manzanillo era utilizado con frecuencia en el campo, llegando a aplicarse en algunas ocasiones con fines criminales; además de acarrear graves consecuencias cuando era ingerido como alimento por determinados animales. En este sentido, recuerda que a esta planta se le había achacado erróneamente ser la causa de la ciguatera, (una intoxicación grave causada por la ingestión de ciertos peces) asunto que fue aclarado oportunamente por el naturalista Felipe Poey en su memoria sobre esta enfermedad. (4).

Aparte de describir los caracteres físicos y las propiedades del látex del manzanillo, el autor practicó investigaciones toxicológicas por medio de necropsias, llevó a cabo análisis químicos y observaciones fisiológicas. Gracias a ello pudo demostrar convincentemente la toxicidad del jugo de esta planta. Esto facilitaba la administración de justicia, pues a través de los exámenes toxicológicos propuestos se podía detectar la presencia del manzanillo en los cadáveres.

Otra de las plantas que llamó la atención en esta institución fue Datura arbore L (campana). Sobre la misma fue publicado en 1910 un interesante estudio por el doctor Juan Santos Fernández, a través del cual determinó, después de varias observaciones, que provocaba la midriasis monocular —dilatación excesiva de la pupila, con inmovilidad del iris. (5).

En este trabajo manifestó la expectación que le habían causado dos casos, que de forma casual habían adquirido esta afección al tener contacto con la planta. Pudo confirmar el diagnóstico que ya se había formado, induciendo el contacto de otras personas con la referida planta. El hecho de que el doctor Santos Fernández hubiese verificado que una planta como ésta, que era tan común en los jardines de Cuba, fuese capaz de causar daños a la salud, constituía una valiosa información encaminada a prevenir a la población de este peligro.

Un año después, el doctor Gastón Alonso Cuadrado, químico distinguido de la Academia (6), disertó sobre el análisis químico del agua de coco (Coco nucifera L.). En su exposición dio a conocer que esta indagación estuvo motivada por el interés de su amigo, el doctor Valdés Anciano, de conocer si el agua de coco contenía albúmina vegetal, por lo que se dio a la tarea de analizar los componentes químicos de la misma. En este 
estudio detalla los análisis realizados en frutas adquiridas en el mercado, con el objetivo de esclarecer la composición de dicho líquido, para lo cual llevó a cabo numerosos experimentos que demostraron que el agua de coco no contiene materia proteica apreciable, pero sí cloruro de sodio, sulfato de alúmina y glucosa (7).

Este trabajo, el cual el propio autor no consideró profundo ni completo, recibió una gran acogida entre los miembros de la Academia, ya que demostraba que la acción diurética del agua de coco se debía a los componentes ya señalados y no a la existencia de principios nitrogenados, como se creía desde hacía mucho tiempo por las personas que la utilizaban con este fin. Estos resultados despertaron el interés de los académicos Juan Santos Fernández, Tomás Vicente Coronado y Francisco Héctor Fernández, los cuales solicitaron al doctor Alonso que completara el análisis, pero no sólo del agua de coco en distintos períodos de madurez, sino también del fruto mismo (8).

Como bien se puede apreciar, los trabajos de botánica médicofarmacéutica hasta ahora analizados, se caracterizaban por la aplicación de la observación como procedimiento fundamental, aunque en algunos de ellos era evidente la influencia de la fitoquímica. Otra de las peculiaridades de este período está dada por la escasez de estudios en este sentido, lo que al parecer pudo estar determinado por la ausencia de un plan que sistematizara y organizara los mismos; a pesar de encontrarse respaldados por el capítulo 1, artículo 2 del Reglamento de la institución aprobado en 1904, donde se disponía:

Contribuir al progreso de las ciencias expresadas en su denominación, a) De cuanto sea preciso para el esclarecimiento de la Fauna, Flora y Topografía Médica de Cuba. (9).

Otra de las causas que posiblemente gravitó sobre el desarrollo de estos estudios, pudieran ser las innumerables ocupaciones que tenía que enfrentar la Comisión de Farmacia y Botánica de la Academia, como eran los análisis y comprobaciones de numerosos preparados farmacéuticos que se ponían bajo su consideración, tarea que en gran medida se fue tornando complicada. De igual forma, durante estos años la institución se vio enfrascada en otras no menos importantes responsabilidades, como fueron las cuestiones acerca de la legislación farmacéutica. Sin lugar a dudas, las razones expuestas pueden explicar la escasez de estudios acerca de la materia médica vegetal, que llegaron casi a desaparecer; no obstante, en 1918 se reanudó la publicación de trabajos, con el discurso de recepción 
como académico de número del doctor en Farmacia José Agustín Simpson, quien presentó una memoria acerca de Phaseolus lunatus L. (frijol de Birmania). Simpson relata casos de accidentes por intoxicación ocurridos por la ingestión de estos frijoles en Puerto Rico, razón por la cual fueron iniciadas investigaciones en ese país y a su vez recibidas indicacionés del cónsul de Cuba sobre la necesidad de indagar acerca de los frijoles que se consumían en Cuba. El trabajo desarrollado se caracterizó por la descripción de los caracteres externos de Phaseolus lunatus L., conocido en el comercio como frijol de Java, de Birmania, de Rangoon, Lima, etc., el cual se encontraba en el comercio en considerable diversidad de colores y mezclado con otros granos. Simpson especificó que el ácido cianhídrico, que se encuentra en las variedades más tóxicas, se puede localizar en iguales cantidades en otras plantas como la yuca agria, el laurel cerezo, las almendras amargas y en menor proporción en el humo del tabaco.

Con el objetivo de determinar el grado de toxicidad de estos frijoles, de acuerdo con el contenido de esta sustancia, Simpson desarrolló una serie de análisis químicos y exámenes histológicos, de los cuales obtuvo como resultado que en la variedad denominada Rangoon blanca se encuentra el mencionado ácido en mayor cantidad (10). Al respecto declaraba el autor en sus conclusiones:

«Se han practicado más de 80 análisis sobre las distintas variedades de este grano sin haberse encontrado en ninguna a excepción de la primeramente mencionada cantidad mayor de 8 miligramos por 100 de producto" (11).

Correspondió al doctor Gastón Alonso Cuadrado el discurso de contestación a la memoria antes citada, en el cual resalta la importancia de los resultados científicos obtenidos, al expresar:

La demostración de la producción del ácido cianhídrico en la legumbre llamada "Phaseolus lunatus» es uno de los trabajos de actualidad y de conveniencia para la salud pública, y ha desentrañado el problema de las dificultades que los intereses comerciales, sobre todo si son extranjeros, crean siempre a la Secretaría de Sanidad (12).

... el discurso de ingreso en esta ilustre corporación del doctor José A. Simpson, tiene el gran mérito de la originalidad y de la oportunidad, tiene el de haber proporcionado a la Secretaría de Sanidad, los datos necesarios para sus órdenes de hacer desaparecer del mercado un alimento tan perjudicial a la salud pública, tiene la importancia de una monografía completa y una contribución al estudio de las ciencias naturales, las cuales son las únicas en el orden del conocimiento, que buscan la verdad por la verdad misma, siguiendo por medio del experimento y la 
observación las leyes eternas que preceden la evolución de la materia y de la energía a través del tiempo y del espacio (13).

Aunque el objetivo de la Academia era la presentación y discusión de trabajos encaminados a comprobar las virtudes medicinales de las plantas, en oportunidades fueron expuestos estudios más generales que, de una forma u otra, trataban aspectos relacionados con esta temática, como es el caso de la memoria del doctor José Francisco Vélez Geografía médico sanitaria del Término Municipal del Mariel, la cual recibió el premio Presidente Gutiérrez en 1923. En esta obra, el autor perseguía como objetivo fundamental el estudio del medio natural y, entre los elementos principales de que trata, está el examen de las especies que conforman la flora medicinal de esta región, sobre las que especifica:

Nosotros, para no hacer interminable este trabajo, nos limitaremos a citar solo algunas de las plantas a las que el vulgo atribuye poderes curativos, muchas de las cuales hemos podido comprobar que no son imaginarias (14).

En efecto, cita plantas medicinales, con su clasificación científica, así como las propiedades medicinales que se les atribuyen. Se limita a declarar que se ha comprobado su acción terapéutica, pero sin mayores precisiones. No obstante, recalca la necesidad de realizar estudios acerca de la flora médica cubana por personal científico y comisiones organizadas que investiguen todo su «inmenso poder terapéutico»; es decir, hace una llamada a que la Academia y demás instituciones contribuyan a una mejor utilización de estas plantas por parte de la población.

Los estudios presentados a partir de 1939 se caracterizan por una mayor aplicación de métodos y procedimientos que iban más allá de la simple observación, es decir, incluían además de análisis químicos, estudios prácticos de experimentación clínica. En 1939, el doctor Manuel García Hernández, farmacéutico y miembro de número de la Academia, sometió a consideración de la misma su trabajo Contenido de vitamina $C$ en las frutas cubanas. García Hernández detalla las técnicas utilizadas para determinar la cantidad de vitamina $\mathrm{C}$ en cada una de las frutas analizadas, así como los resultados obtenidos. Para este estudio utilizó 16 frutas cubanas, entre las que se encontraban los plátanos manzano y Johnson, Musa paradisiaca L.; plátano manzano, Musa sapientum L.; el mamey colorado, Calocarpum sapota Jacq.; papaya amarilla y roja, Carica papaya L.; guanábana, Annona muricata L.; piña blanca, Ananas comosus L.; 
limón, Citrus limonum Risso; coco, Cocos nucifera L.; mandarina, Citrus reticulata Blanco; naranja de china, Citrus sinensis L.; toronja, Citrus paradisi Macf.; anón, Annona squamosa L.; canistel, Lucuma nervosa A.D.C.; guayaba blanca Psidium guayaba L. De ellas, pudo determinar que las más ricas en vitamina $\mathrm{C}$ eran los limones, todas las variedades de naranja y la piña, así como que entre las que menos cantidad contenían se encontraban el coco y los plátanos manzano y Johnson (15).

La amplia y destacada labor científica desplegada por el doctor en Farmacia y miembro de la Academia, José Ignacio Noble Xiqués (16), incluyó estudios dirigidos a comprobar si la aplicación, por la población, de determinadas plantas a la cura de ciertas enfermedades era correcta. Uno de sus primeros trabajos encaminados hacia este objetivo fue el realizado sobre el brazilete Caesalpina bahamensis L. El interés por estudiar esta especie se originó en un viaje a la zona de Santa Clara, donde observó a un campesino que vendía «rajitas» hechas de la madera amarillenta de brazilete, y pregonaba que servían para curar las enfermedades de los riñones. A partir de este momento comenzó Noble su investigación, para lo cual utilizó una muestra de esta planta, pero de la región de Pinar del Río, ya que conocía que el brazilete de esa zona era preferido por presentar «poca corteza y mucho corazón» (17). Con respecto al uso que la población hacía de este vegetal, informa el doctor Noble: «Esta planta se emplea en Cuba desde fecha inmemorial en la medicina popular, en las afecciones renales y hepáticas, produciendo franca diuresis» (18).

De inicio, Noble aceptaba la efectividad de esta planta, por lo que dirigió sus experimentos a comprobar qué tipo de componentes existían en la misma, que hacían posible que se aplicara con buenos resultados en estas afecciones. El procedimiento utilizado perseguía, en principio, precisar si contenía saponina, lo cual pudo verificar, por lo que se justificaba el uso indicado; pero lo curioso en este caso era que se utilizaba correctamente, ya que la parte empleada era la médula y no el líber. Esto llamó la atención del doctor Noble, quien una vez terminado su análisis comentó: «... como humanos, nos llama la atención que el vulgo use de por sí, naturalmente y en forma propia, lo que a vuestro servidor le ha costado tanto trabajo encontrar como conclusión» (19).

Del mismo origen fue otro de sus trabajos, publicado en 1947, en esta oportunidad sobre la papaya Carica papaya L., y su aplicación contra la hipertensión arterial. Expone que se apoyó en la información que brindan los diferentes tratados médicos, los cuales definen que esta planta contiene en sus hojas, un alcaloide llamado carpaína -que tiene propiedades cardiotónicas semejantes al digital-, pero dado que el vulgo lo que utilizaba 
es el fruto, se dio a la tarea de localizar este componente activo en esta parte de la planta, con el propósito de razonar el empleo popular de la misma. En su empeño por alcanzar este objetivo, aplicó una serie de técnicas, que incluían la evaporación, el examen microscópico y el análisis microquímico. Como resultado, pudo comprobar que en el fruto también se puede localizar este alcaloide, aunque en una cantidad tan insignificante que no es recomendable con fines terapéuticos; por lo tanto, la utilización que hace la población de esta fruta contra la hipertensión carecería - según Noble- de respaldo científico; todo lo contrario de su aplicación digestiva, justificada por la presencia de la papayina (20).

En ese propio año, 1947, el doctor Noble publicó un nuevo trabajo, en esta oportunidad acerca de la causticidad del guao Comocladia dentata Jacq. La finalidad de este estudio era identificar el principio cáustico de esta planta, ya que según el propio autor no había sido tratada en ninguna de las obras consultadas por él, tanto cubanas como extranjeras, y por el hecho de pertenecer a la familia anacardiácea debía contener los mismos principios que se describen en otras especies de esta familia. Para obtener la muestra que precisaba, se auxilió de un campesino que le ayudó a distinguir el "guao de costa» del "de sabana" y sobre este último realizó un riguroso examen, por medio del método de observación de la coloración del látex y el análisis químico. El resultado alcanzado arrojó que, en efecto, en el guao se hallan presente el cardol y el ácido anacárdico, compuestos cáusticos que se encuentran en otras especies de la misma familia (21).

En 1948, el doctor en Farmacia y académico de número Juan Embill Bollada, se refirió a un estudio experimental sobre una planta exótica, Larrea divoricata Cav., a la cual le atribuían variadas propiedades para la cura de enfermedades. Esta planta, muy extendida por el sur de los Estados Unidos, América Central y del Sur, pertenece a la familia de las zigofiláceas y despertó el interés de este autor, al conocer de los resultados de su aplicación al alivio de dolores reumáticos, por lo que se dedicó a ensayar procedimientos terapéuticos, en este caso por medio de comprimidos, elaborados con la parte de la planta que consideró más activa. La experimentación clínica la realizó sobre siete casos de neuritis rebelde, con resultados positivos, por lo que llegó a la conclusión que Larrea divoricata Cav. es útil para el tratamiento de dolores reumáticos, inflamaciones del mismo origen, en neuritis ciática, en mialgias traumáticas, tortícolis y artritis dolorosas; además señala que la administración en dosis terapéuticas no ejerce acción nociva sobre los aparatos circulatorio, digestivo y renal, ni muestra acciones secundarias desagradables (22). 
Una vez más disertó Noble sobre propiedades medicinales de las plantas, cuando presentó en el propio año 1949 un estudio bioquímico de la berenjena Solanum melongena L. Para el desarrollo del mismo, tomó como punto de referencia un artículo publicado en 1944 por el doctor Antel H. Raffo, del Instituto de Nutrición de Argentina, donde aconsejaba el uso de la berenjena como modificador hipercolesterínico en los cancerosos, tratamiento que ya había sido incorporado como recurso clínico en la práctica diaria del Instituto de Medicina Experimental de Argentina. Los datos aportados sobre la composición química de esta planta y su capacidad para disminuir el colesterol, despertaron el interés del doctor Noble, quien se dio a la tarea de comprobar la acción activa de la solanina sobre el colesterol, para lo que fue preciso llevar a cabo los análisis microquímicos necesarios. Como resultado, el autor pudo verificar que, en efecto, la solanina se encontraba presente en la berenjena; sin embargo, las pruebas realizadas con el denominado líquido estabilizado de la berenjena sobre el colesterol ofrecieron resultados casi nulos. Este líquido estabilizado, según el propio autor, lo obtuvo mediante el tratamiento de la berenjena con alcohol hirviente, para extraer los principios activos de la planta y destruir los fermentos que alteran el $\mathrm{pH}$ de dicho líquido y los principios activos (23).

Estimulada por las conclusiones presentadas por el doctor Noble, la Sección de Farmacia y Química de la Academia, representada por el doctor Ernesto Trelles Duelo, dio a conocer un estudio titulado Acción colagoga de la berenjena, que se limitaba a exponer los resultados, pero sin esclarecer la acción que sobre el colesterol pudiera o no ejercer (24).

Al parecer, estos análisis fueron los primeros intentos de investigaciones sobre la berenjena en Cuba, los cuales pretendían abrir un camino para su uso terapéutico en el país, como lo expresa el doctor Trelles:

\footnotetext{
"Terminamos nuestra "nota preliminan» estimulando a nuestros clínicos, médicos y farmacéuticos laboratoristas, para que de alguna manera, sigan en Cuba por el sendero siempre fecundo de las investigaciones iniciadas por el Profesor Raffo» (25).
}

Con estos estudios, en la mayoría de los casos se pretendía quizás respaldar o corregir el uso empírico popular, pero además, aprovechar la flora medicinal del país en lugar de los preparados sintéticos, pues como afirmaba el doctor Noble Xiqués: «La industria farmacéutica por la multiplicidad presentativa y la subsecuente prescripción ocasiona más intoxicaciones que curaciones» (26). 
El criterio de evitar la medicación química sintética, mientras fuera posible, estaba sustentado en la opinión de que la bioquímica era una ciencia nueva y complicada, por lo que debía preferirse la utilización de medicamentos naturales, y es precisamente por ello por lo que la Academia dirigía sus esfuerzos a estimular la realización de investigaciones farmacoterapéuticas de plantas que eran empleadas por la población. En esta labor participaban, sobre todo, académicos con un perfil profesional químico-farmacológico. Entre ellos, una de las figuras que mostró mayor interés - como ya se ha podido apreciar - fue el doctor José Ignacio Noble, quien una vez más, en 1950, presentó un estudio sustentado en el análisis y comprobación experimental de las virtudes del mastuerzo Lepidium virginicum L., y su empleo en la hipertensión, motivado por una solicitud que diez años atrás le hiciera el doctor Olivio Lubián, médico de Santa Clara. Noble conocía del empleo de esta planta en la medicina popular y, además, le habían llegado noticias acerca de su aplicación en los trastornos hepáticos y en la hipertensión. Del trabajo publicado se deduce que la primera experiencia por él realizada fue estrictamente personal; presentó una colitis aguda, que desapareció al ingerir una tisana con extracto de esta planta. A partir de ese momento, decidió iniciar estudios acerca de la propiedades químico-terapéuticas de ella. Entre los resultados obtenidos estuvo la detección de sales de potasio y saponina, que propiciaban la acción diurética; también confirmaba la utilidad del mastuerzo en las enfermedades hepáticas y recomendaba que debía tomarse en infusiones frías renovadas cada 24 horas (27).

Una vez concluido su estudio y verificadas las virtudes terapéuticas de esta planta, el doctor Noble Xiqués dice de ella que «es una droga al más bajo precio: gratis» (28).

Uno de los últimos estudios de Noble, publicados por la Academia, correspondió a la interesante comprobación clínica de la utilidad del ajo y la cebolla en casos de hipertensión. Para ello tomó como muestra tintura de ajo, confirmando que contiene azufre y sesquiterpenos con propiedades antisépticas broncopulmonares. Además procuró demostrar las propiedades excito-leucocitarias -leucopoyéticas- del ajo. De igual manera analizó clínicamente sus propiedades diuréticas y su consiguiente acción hipotensora (29). 


\section{NOTAS}

(1) Mencia García, M. (1939): Concurso valioso que aportaron a la materia médica nuevas plantas del Continente Americano, t. 76, p. 371.

(2) Le Roy CASSA, J. (1904): Presentación de un monstruo vegetal, t. 42, p. 95-96.

(3) Fernández Benítez, J. A. (1908): Estudio fisiológico quimico y toxicológico del jugo del árbol del manzanillo, t. 45 , p. 55 .

(4) Poey Aloy, F. (1868): «Memorias sobre la enfermedad siguatera ocasionada por los peces venenosos». Repertorio Físico Natural de la Isla de Cuba, t. 2, p. 421.

(5) SAntos Fernández, J. (1910): Midriasis producida por la campana (Datura arbórea), t. 46 , p. $454-456$.

(6) Alonso Cuadrado, G. (1850-1925): Químico farmacéutico, realizó numerosas investigaciones en la Academia de Ciencias de La Habana. Se destacó por la importancia que le concedió al método experimental en la investigación científica. Se le consideró en su tiempo una autoridad en las ciencias químicas.

(7) - (1911): Análisis inmediato del agua de coco, t. 48, p. 131-134.

(8) Acta de la sesión pública ordinaria del 9 de junio de 1911, t. 48, p. 115-116.

(9) Reglamento de la Academia de Ciencias Médicas, Físicas y Naturales de La Habana (1905). Imprenta La Prueba. La Habana, p. 5.

(10) Simpson López de Villavicencio, J. J. (1918): Estudio sobre el ácido cianhidrico en el Phaseolus-lunatus (Frijol de Birmania), t. 55, p. 250-283.

(11) Ibidem, p. 275

(12) Alonso Cuadrado, G. (1918): Discurso de contestación al de ingreso del Dr. José A. Simpson, t. 55, p. 287.

(13) Ibidem, p. 291.

(14) VÉLEz, F. (1923): Geografía médico-sanitaria del término municipal del Mariel, t. 60, p. 85.

(15) García Hernández, M. (1939): Contenido de vitamina $C$ en las frutas cubanas, t. 76, p. 345-347.

(16) Noble y XIQues, I. F. (1893-?): Doctor en Farmacia, desarrolló una significativa actividad en la Academia de Ciencias de La Habana y otras instituciones farmacéuticas del país como la Asociación Farmacéutica Nacional y el Colegio Farmacéutico de La Habana. Fue miembro también de la American Pharmaceutical Association. Se distinguió por su trabajo en el Comité de Revisión del Formulario Nacional. Publicó importantes trabajos en revistas nacionales y extranjeras.

(17) —— (1945): Brazilete. Estudio farmacéutico, t. 83, p. 280.

(18) Ibidem, p. 281

(19) Ibidem, p. 287.

(20) Noble Xiques, I. F. (1947): Fruta bomba (Carica papaya) en la hipertensión, t. 85, p. 198-203.

(21) _ (1947): Sobre la causticidad e identificación del guao (Comocladia dentada Jacq), t. 86 , p. $99-102$.

(22) Embill Bollada, J. (1948): Estudio farmacológico de la Larrea divoricata, Cav. t. 86, p. $147-150$.

(23) Noble Xiques, I. F. (1949): Berenjena: experiencia y comprobación bioquímica de los resultados clinicos, t. 87 , p. 261-267.

(24) Trelles Duelo, E. (1949): Acción colagoga de la berenjena, t. 87, p. 268-276

(25) Ibidem, p. 275.

Asclepio-II-1991 
(26) Noble Xiques, I. F. (1950): Empleo racional del mastuerzo en la hipertensión, t. 89, p. 4 .

(27) Ibidem, p. 3-10

(28) Ibidem, p. 10

(29) Noble Xiques, I. F. (1954): Estudio dietético, químico y terapéutico del ajo y la cebolla, t. 92, fascículo III, p. 377-386

Todos los trabajos aquí citados fueron publicados - salvo otra indicación- en los Anales de la Academia de Ciencias Médicas, Físicas y Naturales de La Habana, por lo que sólo se brinda el tomo y páginas en que aparecieron. 